\title{
Protocolo de manejo de la vía aérea en pacientes con sospecha o diagnóstico de SARS-CoV-2/COVID-19
}

\section{Airway management protocol for patients with suspected or diagnosed SARS-CoV-2/COVID-19}

\author{
Dr. Jhonn Sebastián Forero-Vega,* Dr. Orlando Carrillo-Torres, \\ Dra. Yessica Tatiana Salamanca-Sana, ${ }^{\S}$ Dra. Verónica Camacho-Vacherón"
}

RESUMEN. En los últimos días se han publicado diversos protocolos para la prevención de la transmisión del virus COVID-19 durante la manipulación de la vía aérea. El artículo describe una forma para el manejo de la vía aérea que es utilizado en algunos hospitales de la zona de Boyacá en Colombia y en la Ciudad de México. Este consta de un total de nueve fases que describen desde la preparación del equipo médico a utilizar, así como del personal médico que realizará el procedimiento, hasta el retiro del tubo endotraqueal. Estas fases son: colocación del equipo de protección personal, preparación, preoxigenación, inducción, intubación, confirmación, sonda de aspiración, extubación y retiro y desecho de material.

ABSTRACT. In recent days, various protocols have been published for the prevention of transmission of the COVID-19 virus during manipulation of the airway. The article describes a form for airway management that is used in some hospitals in the Boyacá Colombia and Mexico City. This consists of nine phases that describe from the preparation of the medical equipment to be used, as well as the medical personnel that will perform the procedure, to the removal of the endotracheal tube. These phases are: placement of personal protective equipment, preparation, pre-oxygenation, induction, intubation, confirmation, aspiration tube, extubation and removal and disposal of material.

\section{INTRODUCCIÓN}

E n los últimos días se han publicado diversos protocolos para la prevención de la transmisión del virus COVID-19 durante la manipulación de la vía aérea en donde se utilizan elementos como acrílicos, mascarillas especiales, posiciones, cuatro manos y/o técnicas de tipo ventilación apnéusica, intubación con fibrobroncoscopio vía nasal o videolaringoscopio. El presente artículo describe el protocolo propuesto para el manejo de la vía aérea que ha sido utilizado hasta este momento en algunos hospitales de la zona de Boyacá en Colombia y en la megalópolis en la Ciudad de México.

Este protocolo se realiza ante la alerta mundial por la pandemia de COVID-19 y teniendo en cuenta que aproximadamente $5 \%$ de los pacientes pueden presentar condiciones críticas que requieren manejo avanzado de la vía aérea ${ }^{(1)}$. Además, los pacientes con esta enfermedad pueden requerir procedimientos quirúrgicos de urgencia que, de igual forma, requieran abordaje de la vía aérea por el anestesiólogo en una sala de quirófano. El protocolo consta de nueve fases principales que iremos describiendo a continuación.

\section{PRIMERA FASE: ELEMENTOS DE PROTECCIÓN PERSONAL (EPP)}

La transmisión del virus se da por aerosoles (Tabla 1) y teniendo antecedentes de brotes similares donde un porcentaje importante de los casos fueron de origen nosocomial, se debe tener en cuenta como primera medida el uso adecuado de todos los elementos de protección personal ${ }^{(2,3)}$.
Anestesiología

Octubre-Diciembre 2020

Vol. 43. No. 4. pp 225-229

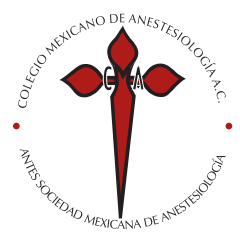

Palabras clave:

COVID-19, vía aérea, manejo, protocolo, Colombia, México.

Keywords:

COVID-19, airway, handling, protocol, Colombia, Mexico.

* Médico Anestesiólogo adscrito al Servicio de Anestesiología del Hospital Regional de

Duitama; Boyacá, Colombia.

‡ Médico Anestesiólogo adscrito al Servicio de Anestesiología del Hospital General de México. CDMX, México.

$\S$ Médico General de la Clínica de Especialidades Sogamoso; Boyacá, Colombia.

" Médico Anestesiólogo adscrita al Servicio de Anestesiología del Hospital General de México «Dr. Eduardo Liceaga». CDMX, México.

Solicitud de sobretiros: Orlando Carrillo-Torres Avenida Cuauhtémoc 590, Int. 204 Narvarte Poniente,

Alcaldía Benito Juárez, Ciudad de México. Tel. 5527892000 ext. 1376 E-mail: orlo_78@hotmail.com

Abreviaturas:

$\mathrm{EPP}=$ Equipos de protección personal. $\mu \mathrm{g}=$ Microgramo. $\mathrm{mg}=$ Miligramo. $\mathrm{kg}=$ kilogramo.

Recibido para publicación: 04-06-2020

Aceptado para publicación: 02-07-2020 
Tabla 1: Procedimientos que generan aerosoles(3).

- Ventilación no invasiva

- Laringoscopía directa y por video

- Intubación traqueal

- Endoscopía y fibrobroncoscopía

- Abordaje quirúrgico de la cara anterior del cuello (traqueostomía, cricotiroidotomía)

- Aspiración traqueal, sin sistema cerrado

- Extubación

- Reanimación cardio-cerebro-pulmonar

Colocación de elementos de protección personal según protocolo institucional que incluya:

- Tapabocas N95.

- Monogafas personales.

- Gorro.

- Uniforme antifluidos institucional.

- Traje de protección de plástico para cuerpo completo.

- Bata antifluidos.

- Careta.

- Botas quirúrgicas.

- Doble par de guantes ${ }^{(2)}$.

\section{SEGUNDA FASE: PREPARACIÓN}

- Sitios específicos para el manejo de pacientes con COVID-19, si el paciente está hospitalizado debe ser en la habitación que se encuentre aislada, si va a quirófano se debe destinar sólo un quirófano para estos pacientes, así como una ruta de acceso y egreso.

- Se debe prever y dar intubación temprana a quien lo requiera.

- Corroborar alergias conocidas y ayuno de ocho horas previo a la intubación (no aplica en pacientes urgentes o emergentes).

- El abordaje de la vía aérea idealmente debe ser realizado por el experto en el manejo de la vía aérea (anestesiólogos, internistas, intensivistas, urgenciólogos); sin embargo, ante la contingencia, se debe capacitar a todo el personal médico para su manejo. Los expertos deben intubar en casos específicos cuando la evaluación de la vía aérea definida tenga predictores de vía aérea difícil: obesidad grado III de la OMS, malformaciones craneofaciales, tumores en cuello, limitación para la apertura oral.

- Se debe reducir al máximo el número de personas que atiendan al paciente, sólo dos personas dentro del sitio de intubación, un médico para abordar la vía aérea y otro médico o enfermera como auxiliar de la vía aérea, deben estar capacitados en la realización de la maniobra de Sellick y BURP, administración de medicamentos y ventilar con dispositivo bolsa mascarilla de ser necesario. Además, un auxiliar circulante fuera de la sala en caso de requerir apoyo (no es necesario que tenga los EPP), éste debe tener la lista de chequeo preintubación (Anexo 1) y el carro paro y vía aérea cercanos ${ }^{(4)}$. Las órdenes deben darse clara y efectivamente. Con indicaciones que se verifiquen en todo momento por el receptor, repitiéndolas en voz alta.

- Definir el equipo que se va a usar para el procedimiento, sólo ese equipo ingresará a la sala de intubación y será acomodado de acuerdo con el orden de su uso.

- Tubo de calibre escogido, debe lubricarse en el interior y dejarse guía en forma de palo de golf.

- Laringoscopio o videolaringoscopio de contarse con él

- Equipo de aspiración con sonda.

- Monitorización básica, pulsioxímetro, tensiómetro, electrocardiografía, capnografía.

- Verificación de máquina de anestesia y/o equipo de ventilación y con el sistema de aspiración funcionando.

- Posicionar al paciente según la forma requerida, se puede colocar una rampa para paciente obeso y almohada desechable en caso de laringe anterior ${ }^{(5)}$.

\section{TERCERA FASE: PREOXIGENACIÓN}

- Debe realizarse acorde al estado respiratorio y hemodinámico.

- Instalar entre la mascarilla y dispositivo de ventilación un filtro hidrófobo de alta eficiencia.

- Colocar un plástico transparente entre circuito y máscara para disminuir aún más aerosoles (Figura 1). Este plástico debe ser correctamente adherido o fijado a la mesa quirúrgica, o cama del paciente, para evitar que se desplace mientras se está realizando el procedimiento ${ }^{(6)}$.

- Se debe preoxigenar por cinco minutos con mascarilla facial que haga adecuado sello, flujo de oxígeno a cinco litros, si es requerido por el estado del paciente incrementar el flujo hasta 12 litros, tener en cuenta que a mayor flujo de oxígeno mayor riesgo de producir microgotas; no dar presión positiva si ésta no es necesaria. Realizarla con bajo volumen y mayor frecuencia.

- Usar técnica a dos manos con cualquiera de las dos formas de agarre para evitar fugas ${ }^{(5)}$ (Figura 2). Mantener al paciente en posición a 45 grados y cambiar la posición a necesidad cuando pierda la conciencia. Si el paciente está extremadamente ansioso administre una dosis de midazolam previa de 1-2 mg.

\section{CUARTA FASE: INDUCCIÓN}

- Tener los medicamentos rotulados cargados en sus jeringas desde antes de la preoxigenación. Se debe practicar 


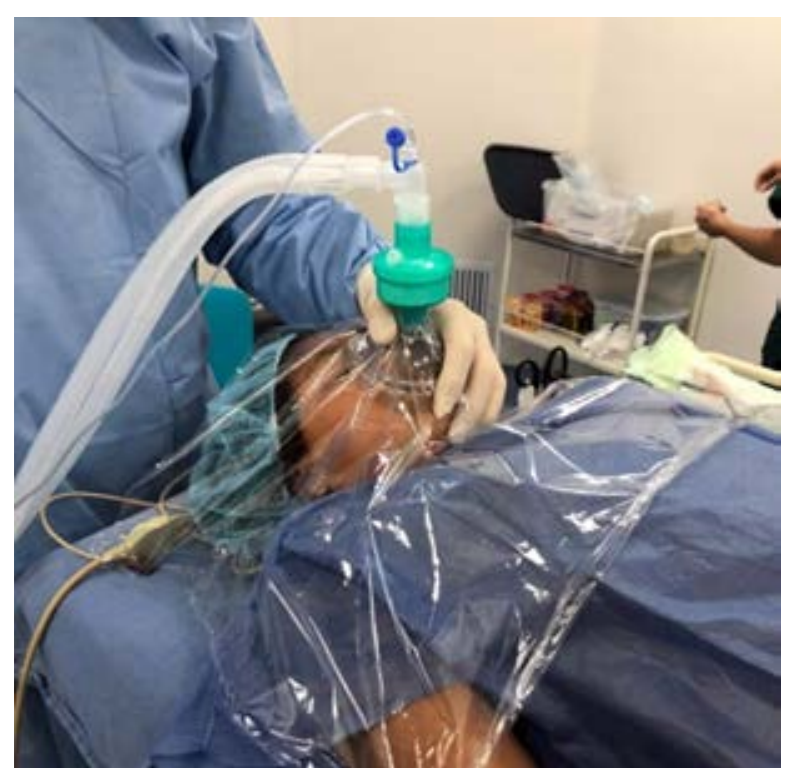

Figura 1: Filtro hidrófobo y mascarilla transparente para la ventilación.

inducción de secuencia rápida en todos los escenarios $(\text { Tabla 2) })^{(6)}$.

- Si el paciente presenta inestabilidad hemodinámica no se debe usar propofol como primera línea (cambiar a midazolam, ketamina o etomidato dependiendo el contexto); además, se debe prever el utilizar vasopresores para el manejo de hipotensión.

\section{QUINTA FASE: INTUBACIÓN}

- De tener entrenamiento en el manejo del videolaringoscopio, úselo; de no ser así realice laringoscopía convencional.

- Mantenga su cara lo más lejos posible de la vía aérea del paciente. Aboque el tubo a la glotis y vaya retirando la guía mientras introduce el tubo, deje el tubo en la distancia que se va a fijar e insufle inmediatamente el neumotaponador, conecte el filtro al tubo directamente y luego al circuito (Figura 1). Empaque inmediatamente el laringoscopio como objeto altamente contaminante.

- Se contraindica el uso de fibrobroncoscopio como primera línea en el manejo de la vía aérea difícil por riesgo de contaminación; sin embargo, si con el uso de videolaringoscopio no se consigue intubación se puede practicar fibrobroncoscopía transnasal.

- La mascarilla laríngea sólo debe ser usada en caso de no lograr intubación, no se recomienda su uso para procedimientos quirúrgicos en estos pacientes ${ }^{(7)}$.

- Abordaje anterior de la vía aérea.
De ser necesario se recomienda técnica de bisturí, bujía y tubo endotraqueal, no usar técnica de catéter con ventilación en jet por riesgo de formación de aerosoles.

\section{SEXTA FASE: CONFIRMACIÓN DEL TUBO}

- Se recomienda confirmación con capnografía; si no se cuenta con ésta proceder a auscultación en tres focos sobre la bolsa plástica y visualización de elevación simétrica del tórax.

\section{SÉPTIMA FASE: SONDA ORO/NASOGÁSTRICA}

- Se debe colocar de inmediato sonda orogástrica o nasogástrica para disminuir la necesidad de contacto con la vía aérea $^{(4)}$.

\section{OCTAVA FASE: EXTUBACIÓN O TRASLADO DE PACIENTE INTUBADO}

- Evite desconectar del circuito dentro de lo posible, si necesita desconectarlo para trasladar al paciente o cam-

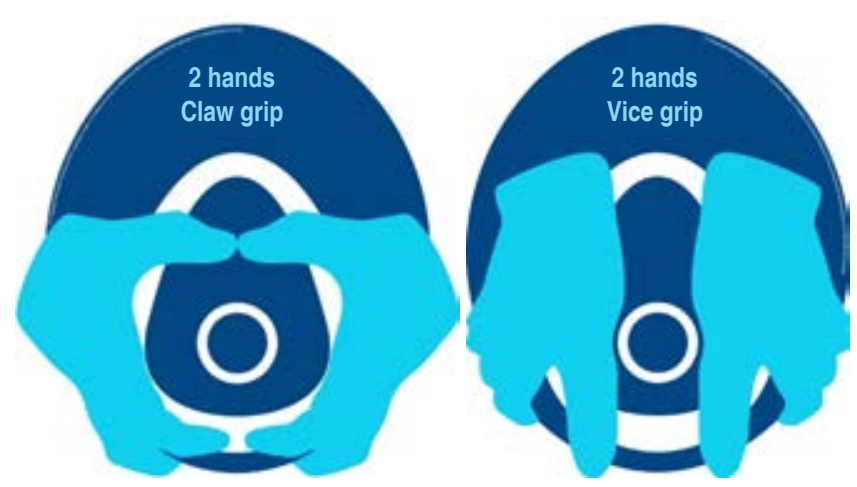

Figura 2: Técnica a dos manos para evitar salida de micropartículas ${ }^{(5)}$. Modificado de Higgs A.

\section{Tabla 2: Fármacos utilizados para inducción rápida.}

\begin{tabular}{ll}
\hline Fármaco y dosis & Secuencias de uso \\
\hline Lidocaína $1 \mathrm{mg} / \mathrm{kg}$ & a. Fentanilo-propofol succinilcolina \\
Fentanilo $2-3 \mu \mathrm{g} / \mathrm{kg}$ & b. Fentanilo-midazolam-succinilcolina \\
$\begin{array}{c}\text { (administrar lento) } \\
\text { relajantes musculares: }\end{array}$ & c. Fentanilo-rocuronio-propofol \\
- Rocuronio $1.2 \mathrm{mg} / \mathrm{k}$ & d. Fentanilo-midazolam-rocuronio \\
- Succinilcolina $1 \mathrm{mg} / \mathrm{kg} ;$ & \\
inductores: & \\
- Propofol 1-2.5 mg/kg, & \\
midazolam $150-500 \mu \mathrm{gg} / \mathrm{kg}$ &
\end{tabular}


biar a otro ventilador mecánico siempre debe ocluir el tubo con la pinza.

- Se recomienda extubarlos con la mascarilla facial de preoxigenación puesta, evitar que el paciente tosa, cambiar tan pronto sea posible a mascarilla de no reinhalación y cubrirla con tapabocas.

- Desechar inmediatamente tubo, filtro y circuito anestésico usado como altamente contaminante ${ }^{(4)}$.

- Recuperación.

El paciente debe mantenerse en la sala para realizarse el proceso de recuperación en compañía del mismo personal que abordó la vía aérea.

\section{NOVENA FASE: RETIRO DE EPP DE ACUERDO CON LA GUÍA INSTITUCIONAL}

- Tener en cuenta precauciones, ya que este momento tiene alto riesgo de contaminación.

Nota adicional: si el paciente con COVID-19 requiere un procedimiento quirúrgico no considerado como una urgencia, éste debe ser postergado el mayor tiempo posible hasta no considerarse infeccioso.

Asimismo, es recomendable realizar anticipadamente prácticas deliberadas de la colocación y retiro del equipo de protección antes de estar expuesto. Así como crear escenarios de simulación para el manejo de la vía aérea en estos pacientes ${ }^{(8)}$.

\section{REFERENCIAS}

1. Wu Z, McGoogan JM. Characteristics of and important lessons from the coronavirus disease 2019 (COVID-19) outbreak in China summary of a report of 72,314 cases from the chinese center for disease control and prevention. JAMA. 2020; 323 (13): 1239-1242. doi: 10.1001/jama.2020.2648.

2. Bowdle A, Munoz-Price S. Preventing infection of patients and healthcare workers should be the new normal in the era of novel coronavirus epidemics. Anesthesiology. 2020; 132: 1292-1295. doi: https://doi.org/10.1097/ ALN.0000000000003295.

3. Judson SD, Munster VJ. Nosocomial transmission of emerging viruses via aerosol-generating medical procedures. Viruses. 2019; 11 (10): 940. doi: 10.3390/v11100940.

4. Brewster DJ, Chrimes NC, Do T, Fraser K, Groombridge CJ, et al. Consensus statement: safe airway society principles of airway management and tracheal intubation specific to the COVID-19 adult patient group. Med J Aust. 2020; 212 (10): 472-481. https://www.mja.com.au/journal/2020/ consensus-statement-safe-airway-society-principles-airway-managementand-tracheal.

5. Higgs A. Guidelines for the management of tracheal intubation in critically ill adults. British Journal of Anaesthesia. 2018; 120 (2): 323-352.

6. Meng L, Qiu H, Wan L, Ai Y, Xue Z, Guo Q, et al. Intubation and ventilation amid the COVID-9 outbreak wuhan'experience. Anesthesiology. 2020; 2020; 132: 1317-1332. doi: https://doi.org/10.1097/ALN.0000000000003296.

7. Chen X, Liu Y, Gong Y, Guo X, Zuo M, et al. Perioperative management of patients infected with the novel coronavirus recommendation from the joint task force of the Chinese Society of Anesthesiology and the Chinese Association of Anesthesiologists. Anesthesiology. 2020; 132: 1307-1316. doi: https://doi.org/10.1097/ALN.0000000000003301.

8. Cook TM, El-Boghdadly K, McGuire B, McNarry AF, Patel A, Higgs A. Consensus guidelines for managing the airway in patients with COVID-19. Anaesthesia. 2020; 75 (6): 785-799. doi: 10.1111/anae.15054.

Anexo 1: Lista de chequeo preintubación en paciente con diagnóstico o sospecha de COVID-19 de la ESE Hospital Regional de Duitama.

\begin{tabular}{|c|c|c|}
\hline \multicolumn{3}{|c|}{$\begin{array}{l}\text { Anexo 1: Lista de chequeo preintubación en paciente con diagnóstico o } \\
\text { sospecha de COVID-19 de la ESE Hospital Regional de Duitama. }\end{array}$} \\
\hline & Sí & No \\
\hline 1. Consentimientos informados firmados & $\square$ & $\square$ \\
\hline 2. Personal médico con todos los elementos de protección & $\square$ & $\square$ \\
\hline 3. Paciente monitorizado & $\square$ & $\square$ \\
\hline 4. Se considera vía aérea difícil & $\square$ & $\square$ \\
\hline 5. Tiene riesgo de broncoaspiración & $\square$ & $\square$ \\
\hline 6. Acceso venoso permeable & $\square$ & $\square$ \\
\hline 7. Tubo orotraqueal listo y probado con guía puesta & $\square$ & $\square$ \\
\hline 8. Cánula orofaríngea & $\square$ & $\square$ \\
\hline 9. Laringoscopio o videolaringoscopio listo y probado & $\square$ & $\square$ \\
\hline 10. Equipo de ventilación listo y funcionando y sin fugas & $\square$ & $\square$ \\
\hline 11. Succión funcionando & $\square$ & $\square$ \\
\hline 12. Equipos de carro de vía aérea completos & $\square$ & $\square$ \\
\hline 13. Medicamentos listos y envasados & $\square$ & $\square$ \\
\hline
\end{tabular}


Anexo 2: Organizador gráfico del protocolo de manejo de la vía aérea en pacientes con sospecha o diagnóstico de COVID-19 en sala de urgencias, unidades de cuidado intensivo, salas de cirugía.

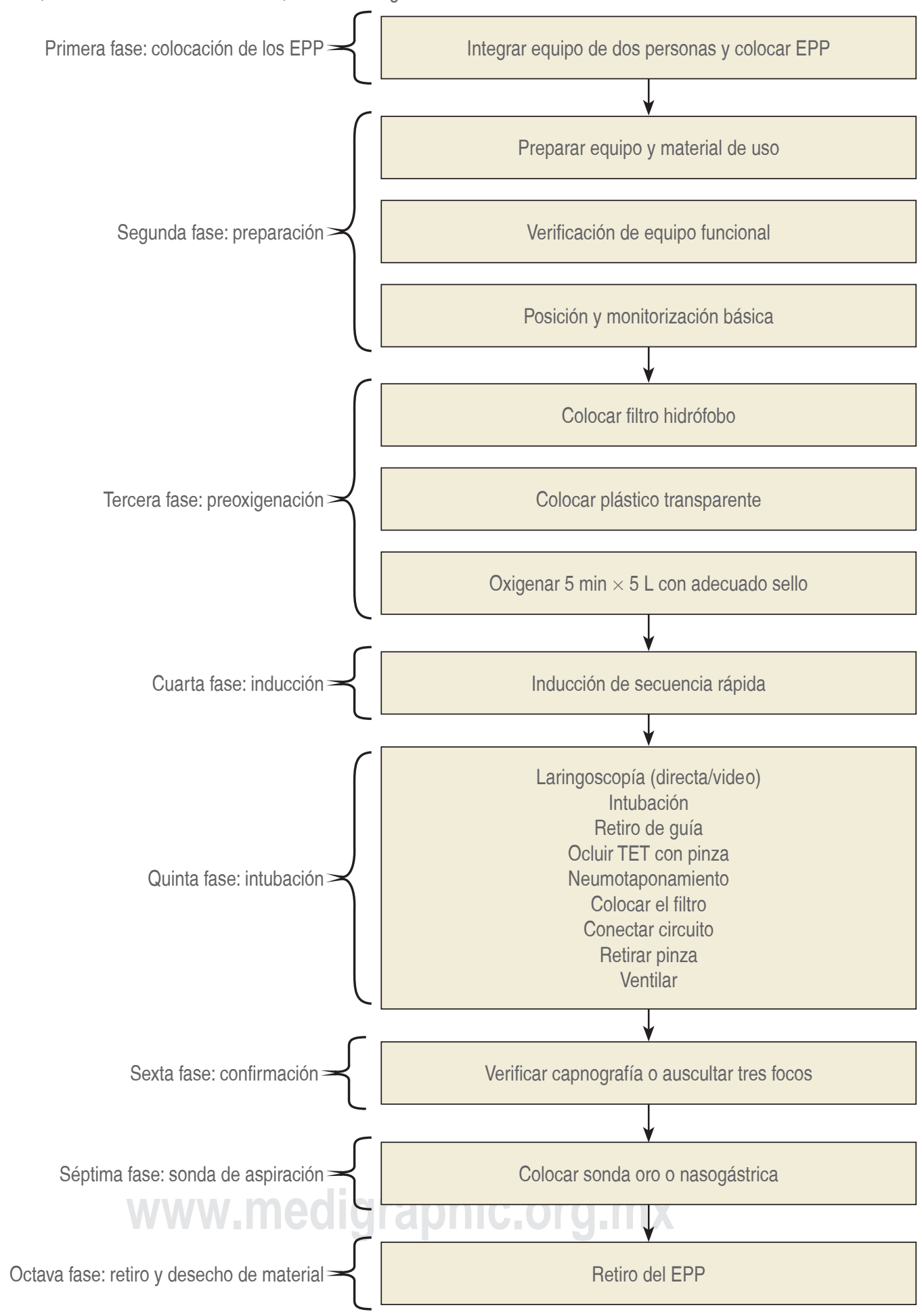

